\title{
PEPTIDE NANOPHOTONICS - FROM BIO-WAVEGUIDES TO INTEGRATED PHOTONIC DEVICES
}

\author{
AMIR HANDELMAN $^{1}$, BORIS APTER ${ }^{1}$, GIL ROSENMAN ${ }^{2}$ \\ ${ }^{1}$ Faculty of Engineering, Holon Institute of Technology, Holon, Israel, handelmana@hit.ac.il \\ ${ }^{2}$ Tel Aviv University, Faculty of Engineering, Physical-Electronics Department, Tel Aviv, Israel
}

\begin{abstract}
Optical waveguiding phenomena in bioinspired chemically synthesized peptide nanostructures can be used to revolutionize emerging fields of precision medical trials and health monitoring. New biomedical light therapy tools and implantable optical biochips can be produced using bioinspired peptide nanostructures due to a unique combination of their intrinsic biocompatibility and remarkable multifunctional optical properties. This essay highlights a new field of peptide nanophotonics. It covers peptide nanotechnology and fabrication processes of peptide integrated optical circuits, basic studies of linear and nonlinear optical phenomena in biological and bioinspired nanostructures, and passive and active optical waveguiding in peptide nanostructures. We show that optical properties of this new generation of biooptical materials are governed by fundamental biological processes. Refolding of the peptide secondary structure is followed by wideband optical absorption and visible tunable fluorescence. In peptide optical waveguides, such a new biooptical effect leads to switching from a passive waveguiding mode in native $\alpha$-helical phase to an active one in $\beta$-sheet phase. Discovery of an active waveguiding effect in $\beta$-sheet fiber structures below the optical diffraction limit opens an avenue for future development of new bionanophotonics devices using ultrathin peptide/protein fibrillar structures for advanced biomedical and nanotechnology applications.
\end{abstract}

Keywords: bioinspired peptide nanostructures, optical waveguides, photonic integrated devices.

\section{INTRODUCTION}

The field of nanophotonics combines fundamental physics, advanced optical materials, and nanotechnology. Optical waveguides (Hunsperger, 2009), which confine and guide light within their structures, are the basic components of integrated chips that can be used in various applications. Such integrated devices that include optical waveguides and combine linear and nonlinear optical and electronic circuits can be used as building blocks of optical communication devices (Prasad, 2004). Medical analysis techniques and disease monitoring are other examples of applications that can benefit from integrated chips that include optical waveguides and photonic devices (Yun and Kwok, 2017).

Nowadays, both biomedical research and clinical research are focused on development of nano- and micro-tools and advanced methods of diagnosis, therapy, and surgery (Vanneman et al., 2012). Photonic technologies are crucial in such new developments for rapid application of the emerging precise light theranostics in medical trials (Humar et al., 2016). Light strongly influences basic biological processes that stimulate application of new biomedical photonic techniques that exploit diverse photochemical, photobiological, and photothermal (Mohammad-Hadi et al., 2018) effects and methods of light-induced therapies, such as photodynamic and optogenetic therapies in addition to traditional optical bioimaging and diagnostics (Paragios et al., 2014).

Here we present a new paradigm of emerging biooptical physics and nanotechnology based on multifunctional bioinspired peptide nanomaterials toward new biomedical nanotherapy tools and implantable integrated optical biochips. Recent research revealed that peptide nanostructures poses unique set of optical properties 
such as nonlinear optical, electrooptical (Gilboa et al., 2017), and optical waveguiding effects (Handelman et al., 2016; Handelman et al., 2018). These newly discovered properties of peptide nanostructures paved the way toward new physics of light propagation in biological and bioinspired nanostructures followed by nanofabrication of optical peptide waveguides. Such nanoscale light waveguides of biological origin can be applied in biomedical theranostics and optical implantable biochips where they are integrated with operating electronics. This new field of peptide nanophotonics (Handelman et al., 2018) is the main subject of my talk.

\section{EXPERIMENTAL}

Light waveguiding is observed in two different regimes passive and active ${ }^{10}$. The passive mode guides the injected light through its optical transmission window and is described by the classical optics of reflection, refraction and diffraction. The key problem in passive optical waveguiding circuits is effective coupling of a light beam to POW. To provide light coupling in our peptide rectangular structures, we designed and fabricated a planar FFF-POW-based Y-splitter for the blue spectral range by incorporating of diffractive grating coupling elements at all of its three ports using FIBtool (Figure 1a,b). The FDTD simulations were performed by the use of the grating coupler realistic 3D model imported to the FDTD simulation software from AFM image data (Figure 1a). Figure 1 demonstrates the experimental results of effective light coupling, waveguiding, splitting and outcoupling under broadband white LED illumination of the input grating.

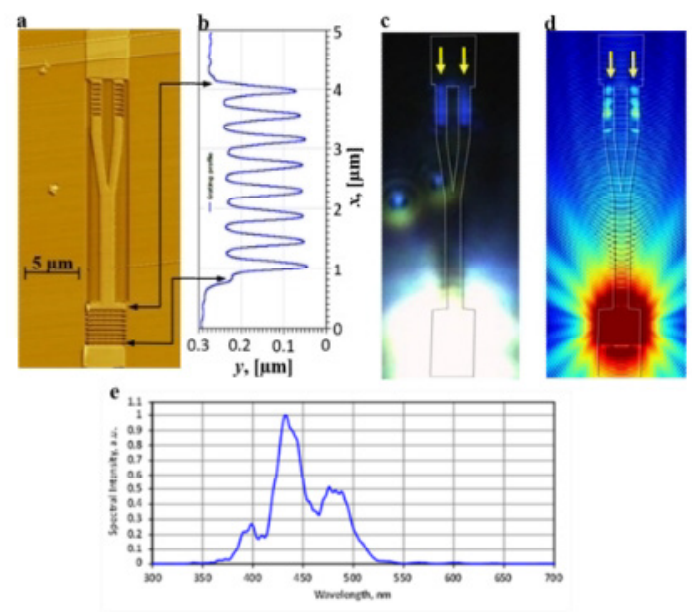

Figure 1. Experimental and simulation observation of the efficient optical coupling, waveguiding and outcoupling in the $1 \times 2$ optical-power Y-splitter under white LED illumination of the input grating. 


\section{RESULTS AND DISCUSSION}

Two elongated, blue light spots observed at the output gratings (Figure 1c) indicate selective wave coupling through grating elements, acting also as an optical filter. The FDTD simulations setup with reproduced experimental conditions shows a good agreement with our experimental data (Figure 1c,d).

\section{CONCLUSIONS}

We introduced all-peptide photonic integrated splitter, composed of bioinspired planar peptide wafers of a large area treated by high resolution patterning. This development opens the avenue for a new field of peptide integrated biooptics where multifunctional peptide optical materials can be fabricated as optical integrated circuits combined with electronic components and interconnections towards lab-on-biochips. Further feature of these peptide structure is their ability to transform from passive to active waveguiding regime in peptide materials by reconfirmation of their secondary structure.

\section{Acknowledgement}

This research was supported by the Ministry of Science, Technology \& Space of Israel.

\section{REFERENCES}

Gilboa, B. et al. (2017), "Strong Electro-Optic Effect and Spontaneous Domain Formation in Self-Assembled Peptide Structures", Adv Sci, 4, 1700052, https://doi.org/10.1002/advs.201700052.

Handelman, A. et al. (2016), "Linear and nonlinear optical waveguiding in bio-inspired peptide nanotubes", Acta Biomater, 30, 72, https://doi.org/10.1016/j.actbio.2015.11.004.

Handelman, A. et al. (2018), "Peptide integrated optics", Adv Mater, 30, 1705776. https://doi.org/10.1002/adma.201705776.

Humar, M. et al. (2016), "Toward biomaterial-based implantable photonic devices", Nanophotonics, 5, 60-80, https://doi.org/10.1515/nanoph-2016-0003.

Hunsperger, R. (2009), Integrated Optics: Theory and Technology, Springer, Berlin, 2009

Mohammad-Hadi, L. et al. (2018), "Photodynamic therapy in 3D cancer models and the utilisation of nanodelivery systems", Nanoscale, 10, 1570, https://doi.org/10.1039/C7NR07739D.

Paragios, N. et al. (2014), Handbook of Biomedical Imaging: Methodologies and Clinical Research, Springer, USA.

Prasad, P.N. (2004), Nanophotonics, John Willey \& Sons, Inc.

Vanneman, M. and Dranoff, G. (2012), "Combining immunotherapy and targeted therapies in cancer treatment", Nat Rev Cancer, 12, 237, https://doi.org/10.1038/nrc3237.

Yun, S.H. and Kwok, S.J.J. (2017), "Light in diagnosis, therapy and surgery", Nat Biomed Eng, 1, https://doi.org/10.1038/s41551-016-0008. 